\title{
Integration of dimension-adaptive obstacle avoidance algorithm in fuzzy-based autonomous vehicle parking
}

\author{
Naitik M. Nakrani \\ Uka Tarsadia University \\ Bardoli, Gujarat \\ India \\ Maulin M. Joshi \\ Electronics and Communication Dept. \\ Gujarat Technological University \\ SCET, Surat, Gujarat \\ India
}

Received: October 5, 2020. Revised: March 27, 2021. Accepted: April 12, 2021. Published: April 16, 2021.

\begin{abstract}
Sensor-based obstacle avoidance and Autonomous vehicle parking have been immensely researched in recent times. An integration of both will increase the usability of autonomous parking systems in dynamic and uncertain environments. The fuzzy logic theory is widely used to learn expert human skills for machines. However, existing fuzzy-based expert systems generally fail to mimic the natural adaptive skills of humans. The expert driver has a natural tendency to adapt to machine dynamics, especially vehicle-related. This paper proposes a novel non-holonomic dimension-based obstacle avoidance parking algorithm that integrates obstacle avoidance capabilities to a standalone parking controller. This algorithm is developed based on adaptive fuzzy membership inferences concerning passenger cars' different sizes and segments. It is tested for various vehicles in simulation results to show the effectiveness of the algorithm.
\end{abstract}

Keywords- Autonomous parking system, Fuzzy Systems and Fuzzy Control, Sensors, Applied system theory.

\section{INTRODUCTION}

$\mathrm{R}_{\mathrm{i}}$ esearch in autonomous parking systems is rapidly increased in the last decade with an increase in advanced automation and robotics. It provides intelligence and safety to human drivers who feel parking a difficult, especially in the case of rear parking. An autonomous parking system gives a vehicle the ability to park itself without any human intervention in any environmental conditions. The majority of work in this domain is related to the static and partially known environment. Practical situations are dynamic and unpredictable, so the systems should be designed with different sensing mechanisms and additional intelligence to make more complex decisions in any circumstances with safety standards. It remains a very crucial challenge to address in commercial autonomous vehicles.

Traditionally an autonomous vehicle parking involves two tasks: path planning and tracking control. Path planning involves joining a feasible path between a start point and an endpoint of parking considering non-holonomic constraints. If it has to be done in a dynamic environment, sensor-based motion planning techniques are used. Tracking control theories are generally fuzzy-based, neural network-based, and nonlinear control system-based methods. A fuzzy-based approach used for parking gives many advantages as it gives a relatively simple, low cost, and computationally efficient design. Also, a fuzzy system can transfer the skills and intelligence to the autonomous vehicles from the expert drivers using linguistics rule-base. In an earlier approach [1], a fuzzy-based intelligent autonomous motion control for parallel parking was presented. A fuzzy behavior-based control system with collision-free maneuver in case of parallel and diagonal parking was given in [2], [3]-[5] developed a linguistic fuzzy-based fifth-order polynomial path generation and tracking method for parallel and garage parking. Another automatic fuzzy-based diagonal 
parking controller was proposed in [6] that performs multitask of deciding driving direction, speed control, and shortest path planning. A skill-based fuzzy logic approach for tight parking space was given in [7]. [8] used a fifth-order polynomial path data along with sensors for training their adaptive neuro-fuzzy control algorithm for parking. A hybrid approach based on FLC, Petri net, and genetic algorithm is proposed in [9] for an automatic parallel parking system. They combined the genetic algorithm and Petri net to determine the optimal path and genetic algorithms to optimize the fuzzy controller's membership function. A similar fuzzy logic-based parking system was also proposed in [10]-[14].

These systems discuss various design and development problems, including trajectory design, steering control, and continuous scanning for dynamicity in the field. According to the literature, scholars have focused on them independently or in groups of many as multitasking. The complex nature of the environment and feedback management remain significant challenges in the development of these systems. Reactive behavior-based fuzzy control architectures for the unknown or dynamic environment can be found in mobile robot navigation problems [15]-[23]. It can be utilized as an integrated part of the vehicle parking system to track any unforeseen obstacle present in the surrounding. In separate studies, [24] proposed an omnidirectional camera-based fuzzy controller automatic parallel parking. [25] also used a CCD vision sensor with the fuzzy system for backing up an autonomous vehicle. Another image-based fuzzy controller for automated car parking systems is developed [26]. The choice of sensors for autonomous vehicles depends upon environmental conditions, the complexity of an algorithm, and computational power availability [27]. A vision-based system provides a bird view, but its algorithm and can be time-consuming. The selection of an ultrasonic sensor may provide a cheaper and accurate solution compared to infrared sensors. In this paper, a group of ultrasonic sensors are used for environmental sensing. formulate a controller's rule base. Machines can easily imitate human intelligence according to fuzzy set theory. For obstacle avoidance navigation, linguistic data from sensors is fuzzified to pick membership functions and values heuristically, either by experiments or expert rules. On the other hand, these fuzzy membership values remain fixed for a particular group of models.

A skilled human driver can navigate between optimum spaces with little awareness about obstacles and a thorough understanding of vehicle dynamics. When driving a compact hatchback or a big Special Utility Vehicle, the drive-through experience is different. Similarly, a fuzzy system with a fixed set of membership ranges may not fit all the cars, i.e., hatchback, sedan, SUV, etc. The multiple vehicles with different dimensions percept small, medium, and far distances in different ways. As the car changes, the fuzzy membership values must be changed, as conceptualized in Fig. 1. (a-c).

This paper contributes a novel vehicle dimension-based obstacle avoidance parking algorithm that employs a parameter derived from the vehicle's actual dimensions that can adapt fuzzy variables membership values of the parking module and effectively parks a car of various sizes. We created a hybrid intelligent autonomous vehicle parking system with an obstacle avoidance module as part of this research. In literature, the control system's behavior is validated by the simulation modelling[28], [29]. This algorithm is also tested for two different segments of the vehicle in a simulated environment.

This paper's layout is as follows: A block diagram of the autonomous parking system is discussed briefly in Section 2 . Section 3 describes the dimension-based obstacle avoidance algorithm is described in detail in Section 3. Different scenarios for two distinct segments of vehicles are simulated in a confined area with moving obstacles in Section 4 to illustrate the proposed algorithm's validity. Finally, the conclusions are presented.

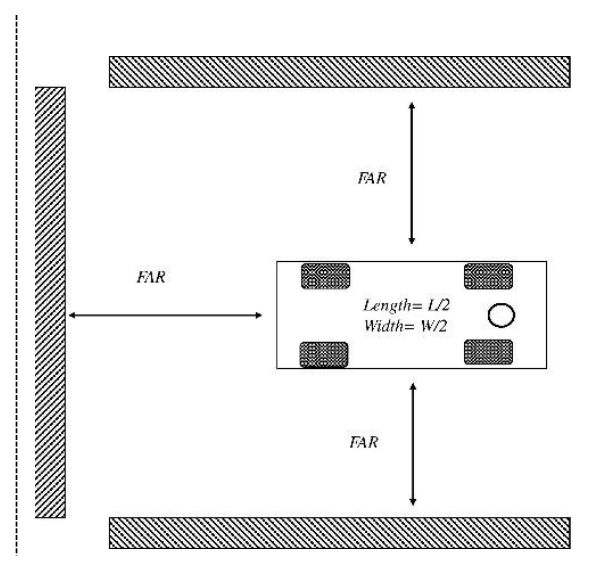

(a) Small size vehicle

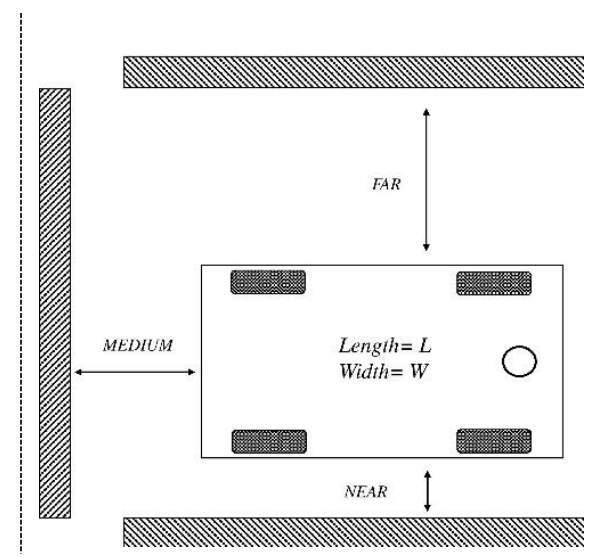

(b) Medium size vehicle

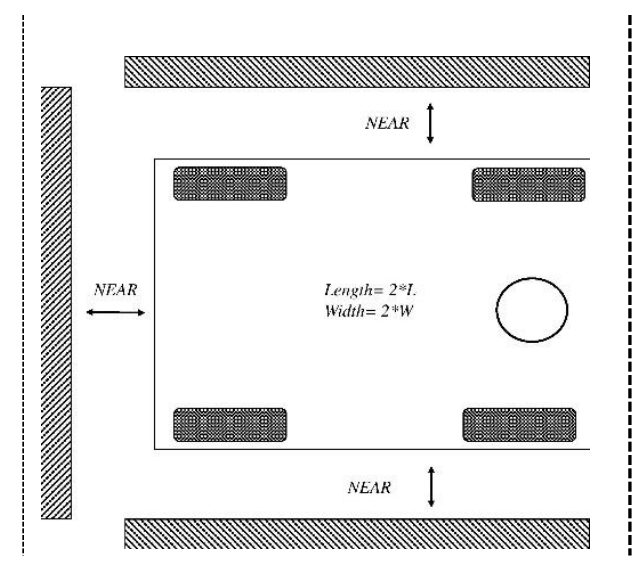

(c) Large size vehicle

Fig. 1. Change in the inference of the environment concerning the size of the vehicle

Numerous algorithms for parking problems are available in the literature to use fuzzy logic theory because of its potential to use linguistic knowledge needed for complex systems to 


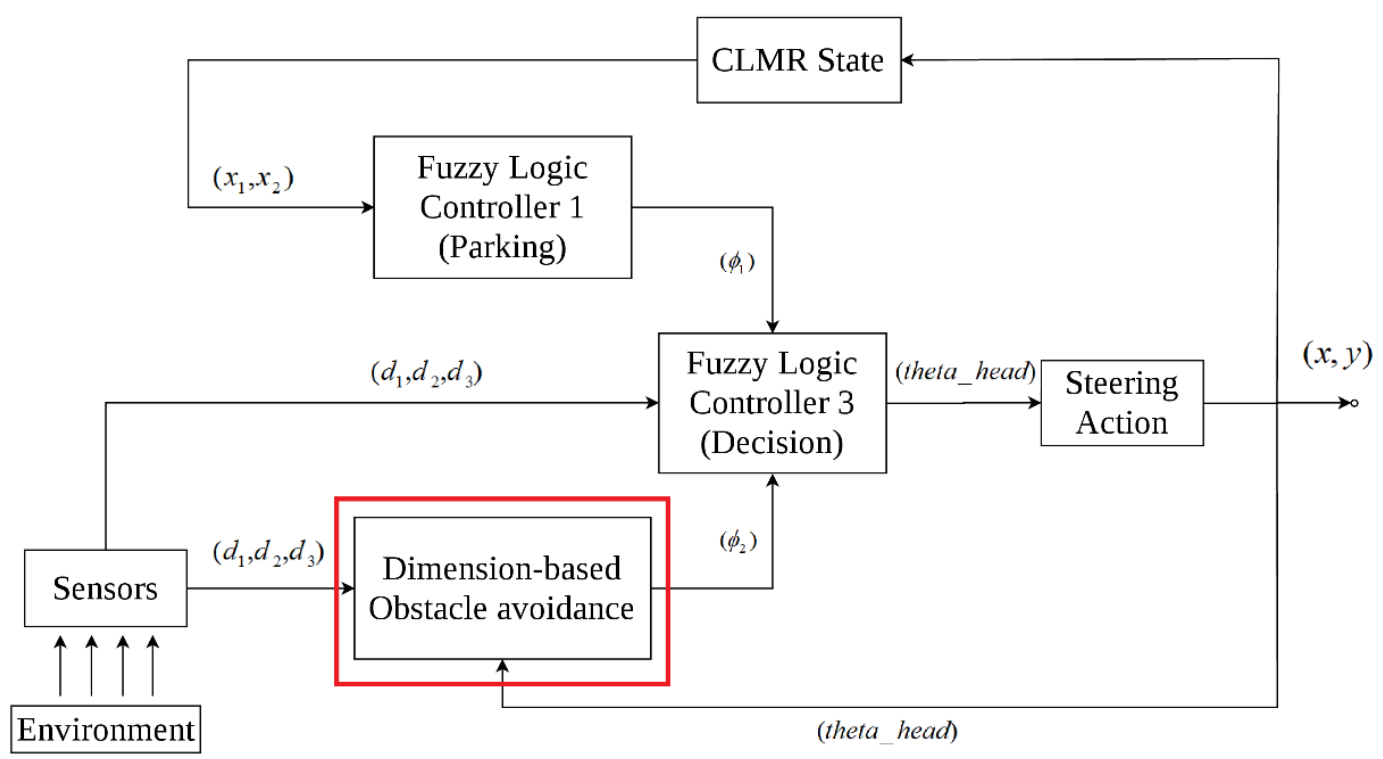

Fig. 2. An architecture of a hybrid autonomous parking system

\section{FUZZY-BASED AUTONOMOUS PARKING ARCHITECTURE}

A hybrid intelligent autonomous parking system architecture is developed, as shown in Fig. 2. It is a multi-level fuzzy logic control architecture. It has two major blocks: one for parallel parking and one for navigation. Third decision controller switches and passes the output of both controllers to the vehicle for the steering action. We utilize the non-holonomic data to convert our navigation controller into a dimension-based obstacle avoidance controller. During parking maneuver, if the obstacles are sensed in a nearby environment, a fuzzy controller called a decision controller would switch Car-like mobile robot's (CLMR) command to the dimension-based obstacle avoidance. The dimension-based obstacle avoidance gives novelty to this work compared to the traditional navigation algorithm found for mobile robots because it is made variable concerning the vehicle's dimension.

\section{A. Parking Controller}

The system shown in the previous section is designed to enable autonomous parking successfully in a dynamic environment without a collision. The system has a standalone design for parallel parking. It is a two-input one output fuzzy logic control. This controller aims to steer the CLMR towards parking space following the fifth-order polynomial path by utilizing basic geometric information provided in Fig. 3.

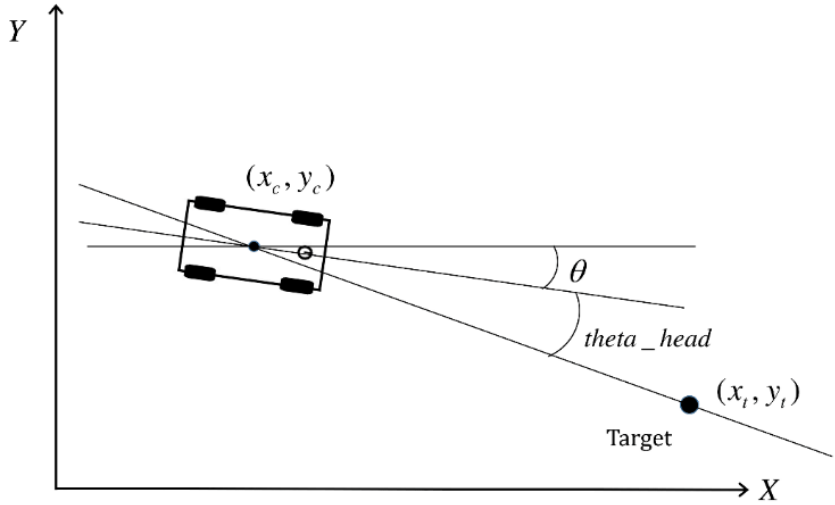

Fig. 3 Geometry used for parallel parking

Let the target location for parking is $\left(\mathrm{x}_{\mathrm{t}}, \mathrm{y}_{\mathrm{t}}\right)$, and the current vehicle state is $\left(x_{c}, y_{c}, \theta\right)$, where $\theta$ is the orientation of the vehicle w.r.t positive $\mathrm{X}$-axis. The angle between vehicle orientation and target location (theta_head) can be defined as per (1),

$$
\text { theta_head }=\tan ^{-1}\left(\frac{y_{t}-y_{c}}{x_{t}-x_{c}}\right)
$$

And the orientation of the vehicle $(\theta)$ concerning the positive $\mathrm{X}$-axis is defined as,

$$
\theta=\tan ^{-1}\left(\frac{y_{c}}{x_{c}}\right)
$$

The parking control design has two inputs $\left(\mathrm{x}_{1}\right.$ and $\left.\mathrm{x}_{2}\right)$ to command the steering angle $(\varphi)$ of CLMR to perform a parking task. The two inputs $\mathrm{x}_{1}$ and $\mathrm{x}_{2}$, are defined as follows:

$$
\begin{aligned}
& x_{1}=\text { theta_head } \\
& x_{2}=\theta
\end{aligned}
$$


The linguistic rules designed for parking mode FLC follow the fifth-order polynomial trajectory between initial and final locations. Such a fifth-order path following is best modeled as a sliding mode line $\left(\mathrm{x}_{1}-\mathrm{x}_{2}\right)$ on which the vehicle follows to reach the goal. When $\left(\mathrm{x}_{1}-\mathrm{x}_{2}\right)$ becomes zero, it indicates that the car is correctly moving towards theta_head. When $\left(\mathrm{x}_{1}-\mathrm{x}_{2}\right)$ is nonzero, appropriate steering action $\varphi$ is taken to converge them to zero. A rule-based is generated based on the fact that both input angles of this fuzzy system remain equal. When the vehicle is near to target, both angles co-aligned with each other horizontally, and it gives the final posture of the car inside the parking space.

\begin{tabular}{|c|c|c|c|c|c|c|c|}
\hline$x 1 \phi^{x 2}$ & NB & NM & NS & ZE & PS & PM & PB \\
\hline NB & ZE & NS & NM & NB & NB & NB & NB \\
\hline NM & PS & ZE & NS & NM & NB & NB & NB \\
\hline NS & PM & PS & ZE & NS & NM & NB & NB \\
\hline ZE & PB & PM & PS & ZE & NS & NM & NB \\
\hline PS & PB & PB & PM & PS & ZE & NS & NM \\
\hline PM & PB & PB & PB & PM & PS & ZE & NS \\
\hline PB & PB & PB & PB & PB & PM & PS & ZE \\
\hline
\end{tabular}

(a)

\begin{tabular}{|c|c|c|c|c|c|c|c|}
\hline$x_{1}{ }^{x}$ & NB & NM & NS & ZE & PS & PM & PB \\
\hline NB & ZE & PS & PM & PB & PB & PB & PB \\
\hline NM & NS & ZE & PS & PM & PB & PB & PB \\
\hline NS & NM & NS & ZE & PS & PM & PB & PB \\
\hline ZE & NB & NM & NS & ZE & PS & PM & PB \\
\hline PS & NB & NB & NM & NS & ZE & PS & PM \\
\hline PM & NB & NB & NB & NM & NS & ZE & PS \\
\hline PB & NB & NB & NB & NB & NM & NS & ZE \\
\hline
\end{tabular}

(b)

Fig. 4 Detailed rule-base for (a) forward parallel parking and (b) reverse parallel parking

Our conceptualization regarding fuzzy membership functions for input and output variables is explained in our previous work [30], [31] with their shapes and threshold values. They are fuzzified into a total of seven different membership functions such as Negative_Big (N.B.), Negative_Medium (N.M.), Negative_Small (N.S.), Zero (Z.E.), Positive_Small (P.S.), Positive_Medium (PM), and Positive_Big (P.B.). As there is a total of 7 membership functions for both input variables, a distinct 49 rules are also determined. Fig. 4 shows detailed 49 fuzzy rules to perform parallel parking in a forward and a reverse manner.

\section{B. Obstacle avoidance controller}

The obstacle avoidance controller is a typical reactive navigation-based architecture. It uses sensed information and navigates through obstacles toward reach goals. It is a fourinput two output fuzzy system. Total eight sensors are used to take distance measurements from nearby obstacles, which then grouped intro 3, left, middle and right, and minimum (each group sensor) three inputs are given to the fuzzy system. The grouping is done depending on forward or reverse motion. The fourth input is the heading angle derived from the target position and current position of CLMR. This controller's output is a change in the heading angle (called the new heading angle). Membership functions of all inputs are shown in Fig. 5. Among four inputs, all left, front, and right sensor inputs membership function is given in Fig. 5(a). The heading angle is fuzzified into Negative, Zero, and Positive membership functions, as shown in Fig. 5(b). The output of a fuzzy system is a new heading angle fuzzified into the same output as the parking controller.

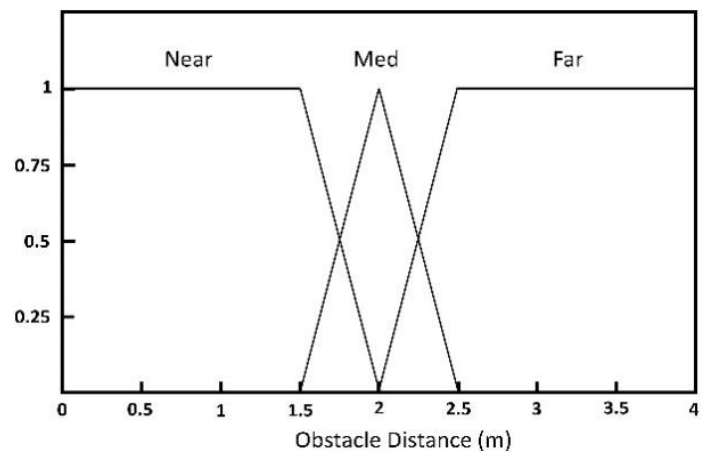

(a)

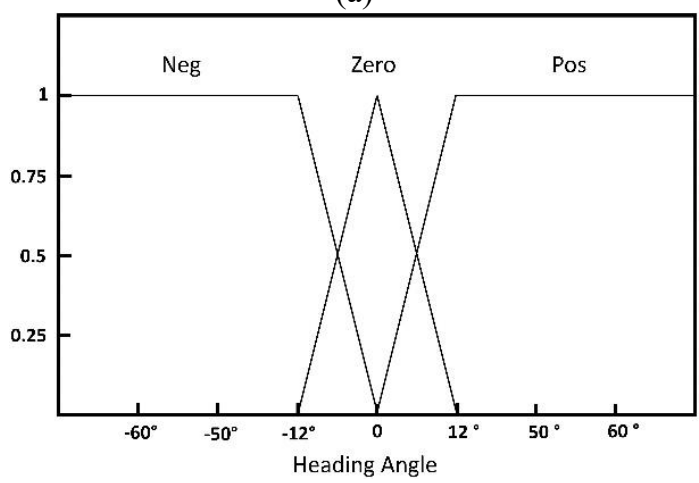

(b)

Fig. 5 Membership Functions of (a) Input: Sensor Distance (b) Input: Heading angle

Based on this input information, the fuzzy rule base is defined to realize various behavior like target steer, obstacle avoidance, edge following, etc. A few samples of these rules are given in Table 1. These fuzzy rules show that the CLMR mainly updates its motion direction and quickly tries to reach the goal if no obstacles are found around the robot. When sensor data indicates that there are obstacles nearby the robot, it must try to change its path to avoid those obstacles. 
Table 1 Fuzzy If-then rules for obstacle avoidance

\begin{tabular}{|c|c|c|c|c|c|c|}
\hline \multicolumn{6}{|c|}{ If } & Then \\
\hline $\begin{array}{c}\text { Rule } \\
\text { no. }\end{array}$ & $\begin{array}{c}\text { Fuzzy } \\
\text { Behaviour }\end{array}$ & $\begin{array}{l}\text { Left } \\
\text { Obs }\end{array}$ & $\begin{array}{l}\text { Front } \\
\text { Obs }\end{array}$ & $\begin{array}{l}\text { Right } \\
\text { Obs }\end{array}$ & $\begin{array}{c}\text { Head } \\
\text { Ang }\end{array}$ & $\begin{array}{l}\text { New } \\
\text { Head } \\
\text { Ang }\end{array}$ \\
\hline 1 & $\begin{array}{l}\text { Target } \\
\text { Steer }\end{array}$ & Far & Far & Far & $\mathrm{N}$ & $\mathrm{Neg}$ \\
\hline 2 & $\begin{array}{l}\text { Target } \\
\text { Steer }\end{array}$ & Far & Far & Far & Z & Zero \\
\hline 3 & $\begin{array}{c}\text { Target } \\
\text { Steer }\end{array}$ & Far & Far & Far & $\mathrm{P}$ & Pos \\
\hline 4 & $\begin{array}{c}\text { Obstacle } \\
\text { Avoidanc } \\
\text { e }\end{array}$ & Near & Near & Far & $\mathrm{N}$ & Pos \\
\hline 5 & $\begin{array}{c}\text { Wall } \\
\text { Following }\end{array}$ & Far & Far & Near & $\mathrm{P}$ & Zero \\
\hline
\end{tabular}

\section{PROPOSED Algorithm}

- Assume a car-like mobile robot (CLMR) with length L and a width of $\mathrm{W}$.

- Let a total of 8 ultrasonic sensors be placed on the robot to sense the surrounding environment, as shown in Fig. 6. These sensors are represented as $\mathrm{S}_{1}, \mathrm{~S}_{2} \ldots \mathrm{S}_{8}$.

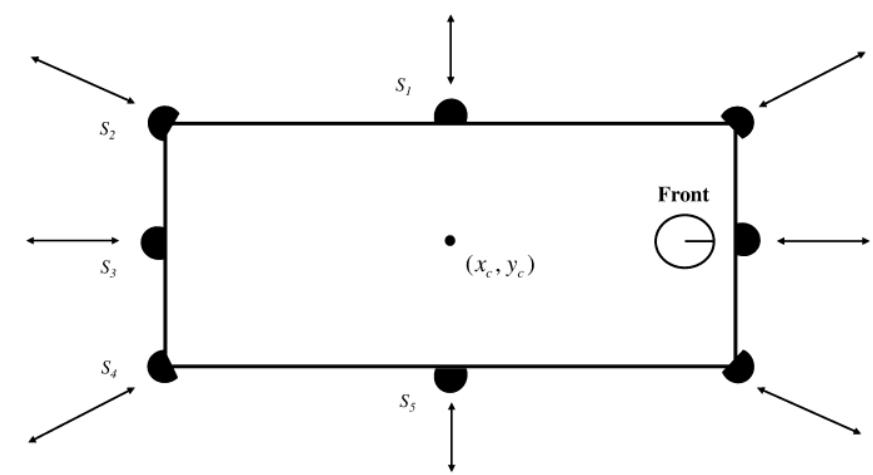

Fig. 6. Placement of ultrasonic sensors on the perimeter of the vehicle

- Let assume the sensors' information is grouped into three different sensor distances, $\mathrm{d}_{1}, \mathrm{~d}_{2}$, and $\mathrm{d}_{3}$, as shown in Fig. 7. Let us presume grouping for reverse parallel parking.

$$
\begin{aligned}
& d_{1}=\min \left\{d i s t_{-} S_{1}, d i s t_{-} S_{2}\right\} \\
& d_{2}=\min \left\{d i s t_{-} S_{2}, d i s t_{-} S_{3}, d i s t_{-} S_{4}\right\} \\
& d_{3}=\min \left\{d i s t_{-} S_{4}, d i s t_{-} S_{5}\right\}
\end{aligned}
$$

- As mentioned below, all three distance groups are further fuzzified into three separate membership functions: Near, Medium, and Far.

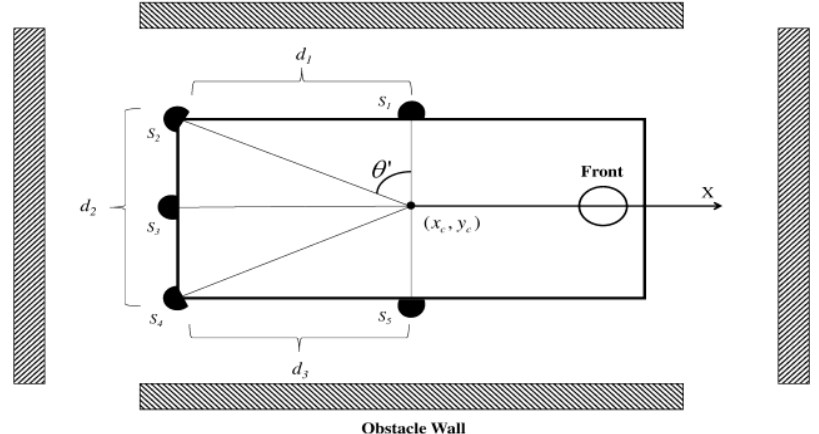

Fig. 7. Ultrasonic Sensors grouping on CLMR for backing up the vehicle

- Their thresholds are calculated as a function of length $\mathrm{L}$, which width $\mathrm{W}$, and are considered constant regardless of vehicle size. These membership functions are defined as:

$$
\begin{aligned}
& \mu_{\text {near }}(x)=\operatorname{trap}\left(\frac{-L}{W}, 0, \frac{L}{W}-\frac{L}{4 W}, \frac{L}{W}\right) \\
& \mu_{\text {med }}(x)=\operatorname{trig}\left(\frac{L}{W}-\frac{L}{4 W}, \frac{L}{W}, \frac{L}{W}+\frac{L}{4 W}\right) \\
& \mu_{\text {far }}(x)=\operatorname{trap}\left(\frac{L}{W}, \frac{L}{W}+\frac{L}{4 W}, \frac{3 L}{W}, \frac{4 L}{W}\right)
\end{aligned}
$$

$$
\text { Where, } x \in\left[0, \frac{3 L}{W}\right] \text { for all the distances }
$$

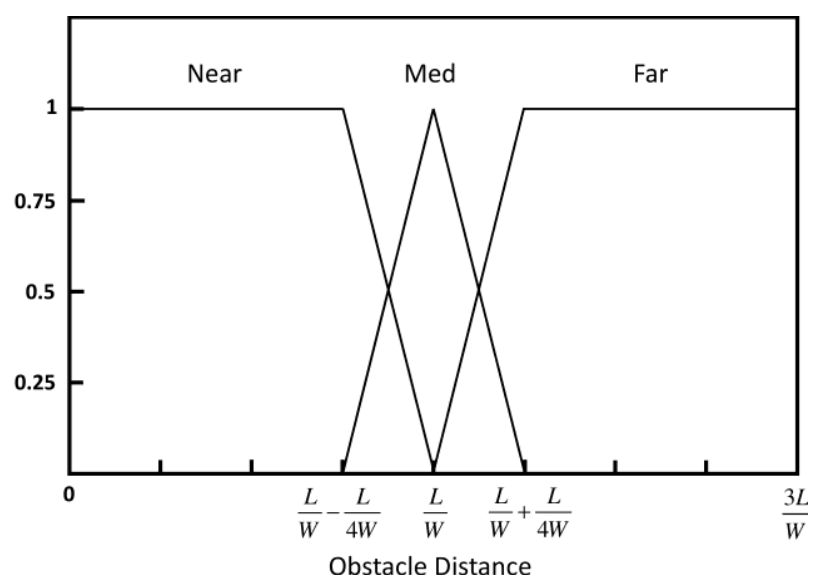

Fig. 8. Modified fuzzy membership function for distances $\mathrm{d}_{1}, \mathrm{~d}_{2}$, and $\mathrm{d}_{3}$.

The shape and value of membership functions are modified for distance $d_{1}, d_{2}$, and $d_{3}$ are shown in Fig. 8.

\section{A. Generalization Steps}

- We define a Constant $\mathrm{K}$, chosen from the actual vehicle dimension and used to adjust fuzzy membership function thresholds that differ with vehicle dimension. The constant $\mathrm{K}$ can be derived from the given length (L) and width (W) as equation (8). 


$$
\frac{W}{2 L} \leq K \leq \min \left(\frac{W}{L}, \frac{L-W}{L+W}\right)
$$

- $\quad$ The factor "K" will be multiplied with a threshold range of fuzzy membership functions using equations (9) - (11),

$$
\begin{aligned}
& \mu_{\text {near }}(x)=\operatorname{trap}\left(a^{\prime}, b^{\prime}, c^{\prime}, d^{\prime}\right) \\
& \quad \text { where, } \\
& a^{\prime}=K^{*} a ; b^{\prime}=K^{*} b ; c^{\prime}=K^{*} c ; d^{\prime}=K^{*} d \\
& \mu_{\text {med }}(x)=\operatorname{trig}\left(a^{\prime}, b^{\prime}, c^{\prime}\right) \\
& =\operatorname{trig}\left(K^{*} a, K^{*} b, K^{*} c\right) \\
& \mu_{\text {far }}(x)=\operatorname{trap}\left(a^{\prime}, b^{\prime}, c^{\prime}, d^{\prime}\right) \\
& =\operatorname{trap}\left(K^{*} a, K^{*} b, K^{*} c, K^{*} d\right) \\
& \quad \text { Where, } x \in\left[0, \frac{3 L}{W} * K\right]
\end{aligned}
$$

- Apply these updated threshold membership functions to the parking of a given CLMR, as seen in Fig. 2.

This algorithm can be extended to any vehicle of any scale and in any complex environment scenario.

\section{Simulation Results}

A parking scenario incorporating a moving obstacle is developed to demonstrate the proposed algorithm's practicality and efficacy. Different parking scenarios for CLMR are considered, indicating the suggested algorithm's potential to perform additional capabilities such as reverse parking, obstacle avoidance, redirection in the event of parking inability, and so on. A parking slot with static and moving obstacles are used to build a Matlab simulation environment ranging 22 by 22 meters. CLMR's speed is assumed to be $1 \mathrm{~m} / \mathrm{second}$, and the vehicle contains ultrasonic sensors mounted on both front and rear sides to detect obstacles. A traveling obstacle's velocity is considered smaller than CLMR's speed.

To illustrate the effect of adding the scaling factor 'K,' two distinct possibilities are considered. Both cases are simulated for various CLMR lengths and widths and various ' $\mathrm{K}$ ' values, with a moving obstacle. The results will demonstrate how CLMR will pass through the narrow pass during parking efficiently and intelligently, which is generally avoided with prefixed membership feature thresholds. Since this algorithm is generalized, it can be used for various sized four-wheel autonomous vehicles.

\section{A. Case 1: Huyndai i20 Length-3996 mm, width- $1734 \mathrm{~mm}$}

To function in the area setup seen in Fig. 9, the L: W ratio is set to balance the actual vehicle axis of the Hyundai i20. Apart from the static obstacles shown as parking space limits, a moving obstacle is considered to be in the direction of the CLMR as it approaches from the opposite end. For a given parallel parking algorithm, CLMR is deemed to be performing a reverse parking technique. Furthermore, the moving obstacle's relative speed is considered to be lower than the CLMR rate for functional purposes.

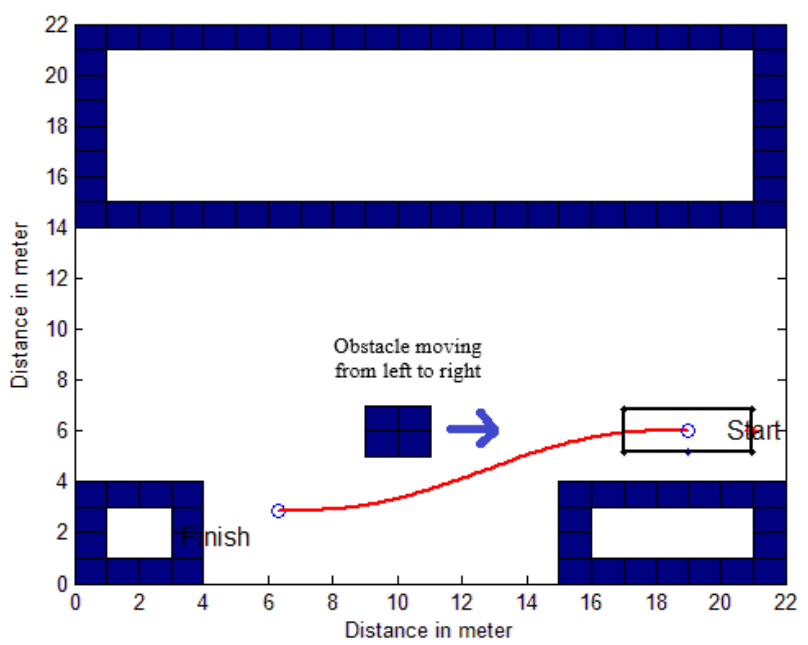

Fig. 9. Scenario indicating the initial and the final locations of CLMR and the initial position and direction of a moving obstacle.

Fig. 9 depicts a typical case scenario with CLMR's initial and final positions and the initial position and trajectory of a moving obstacle. Fig. 10 and Fig. 11 show two different routes, each followed by two CLMRs with varying values of $\mathrm{K}$.

In the first case (having $\mathrm{K}$ equals 1), fuzzy variable membership thresholds are set as per (5), (6), and (7). Fig. 10(ab) shows CLMR entering parking (moving left). Simultaneously, a moving obstacle (moving right) is getting closer to CLMR. Fig. 10(c) depicts the moment when a moving object blocks CLMR's route and, with no other choice, CLMR begins moving correctly (start of obstacle avoidance behavior). CLMR successfully avoids obstacles in Figure 10(d-f). CLMR eventually hits the target parking position after resuming reverse parking, as seen in Fig. 10(g-i).

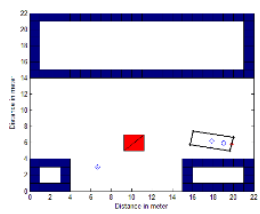

(a)

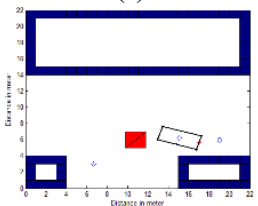

(d)

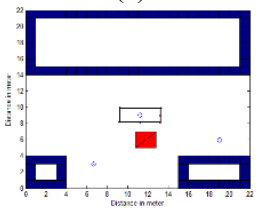

(g)

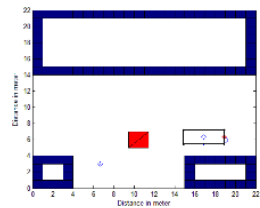

(b)

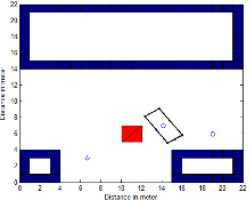

(e)

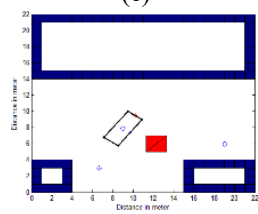

(h)

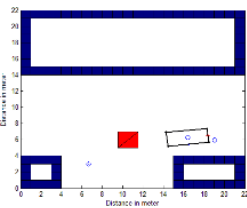

(c)

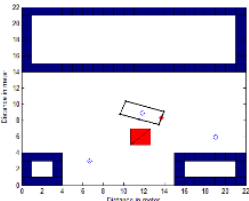

(f)

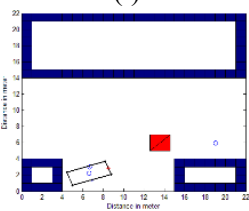

(i)
Fig. 10. The step sequence frames of reverse parking with $\mathrm{K}=1$, no scaling 
Fuzzy variable membership thresholds are set as per (9), (10), and (11) in the second case ( $\mathrm{K}$ is set to 0.25). CLMR attempts to detour the appropriate route when parking with these values, and the inference is modified. CLMR is approaching parking in this situation, as seen in Fig. 11(a-b) (moving left). Simultaneously, a moving obstacle (moving right) is getting closer to CLMR. Fig. 11(c) depicts the moment when a moving object attempts to obstruct the road, but CLMR still finds enough room to navigate between the moving obstacle and the wall (target steering behavior). CLMR is successfully maneuvered in Fig. 11(d-f) while still under the moving obstruction's control.

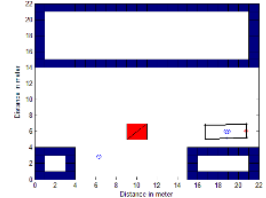

(a)

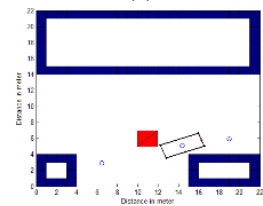

(d)

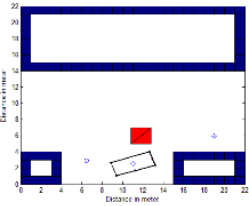

(g)

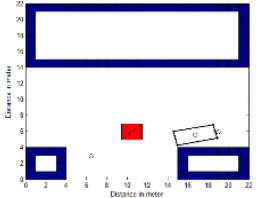

(b)

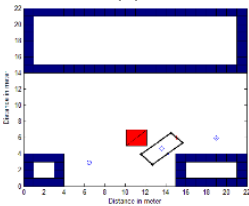

(e)

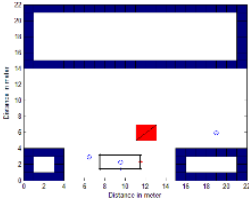

(h)

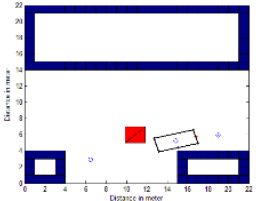

(c)

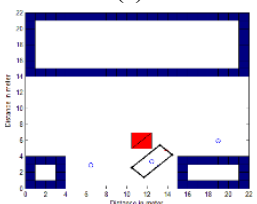

(f)

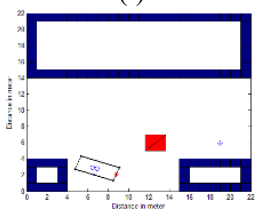

(i)
Fig. 11. The step sequence frames of reverse parking with $\mathrm{K}=0.25$

\section{B. Case 2: Hyundai Verna Length-4440 mm, width-1729 $\mathrm{mm}$}

To function in the environment setup seen in Fig. 12, the L: $\mathrm{W}$ ratio is adjusted to suit Hyundai Verna's actual vehicle measurements. To assess the result for a sedan car, all of the environmental parameters used in this case are identical to those used in the Hyundai 120 scenario. Fig. 12 depicts a typical case scenario with CLMR's original and final positions, as well as the initial position and trajectory of a moving obstacle.

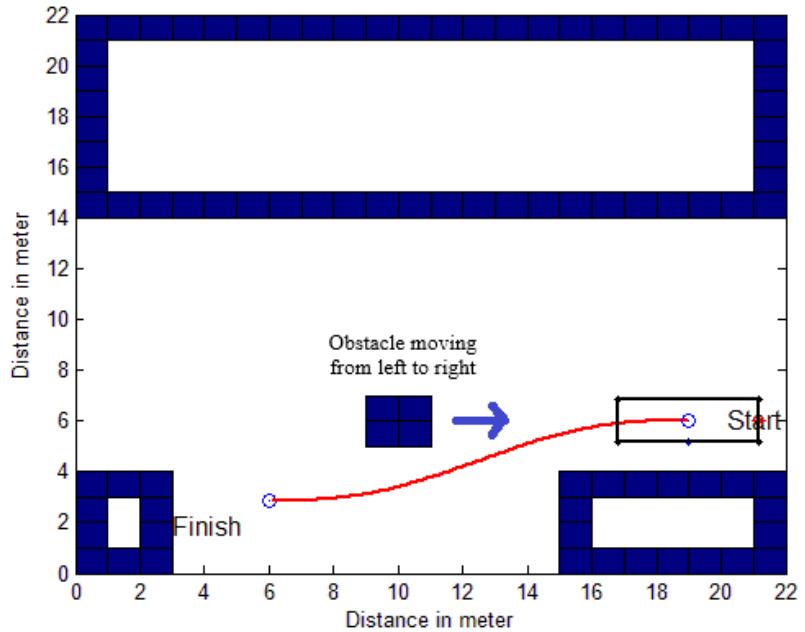

Fig. 12. A scenario indicating the initial and the final locations of CLMR and the initial position and direction of a moving obstacle

The fuzzy variable membership thresholds are set as seen in (5), (6), and (7) in the first case (with $\mathrm{K}$ equal to 1). The inference of fuzzy thresholds is large in the Hyundai Verna due to its larger dimensions than the Hyundai i20. As a result, CLMR follows a path that avoids moving obstacles while maintaining a higher safety margin. The CLMR steering behavior is shown in a step-by-step sequence Fig. 13(a-i).

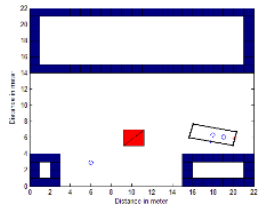

(a)

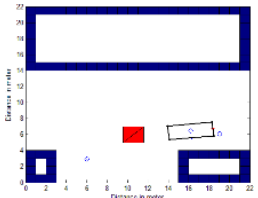

(d)

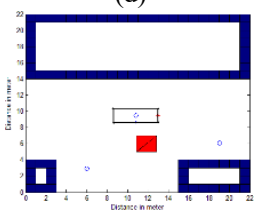

(g)

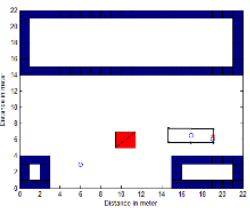

(b)

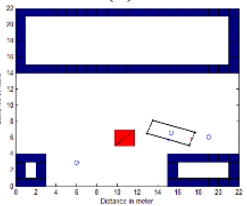

(e)

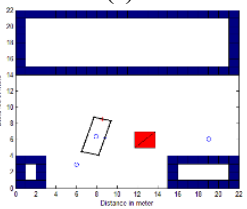

(h)

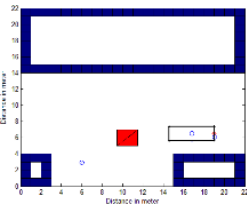

(c)

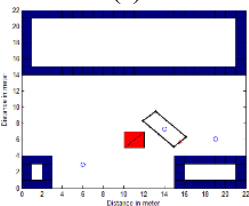

(f)

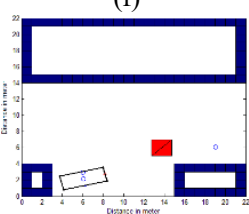

(i)
Fig. 13. The step sequence frames of reverse parking with $\mathrm{K}=1$, no scaling

Fuzzy variable membership thresholds are set as per (10), (11), and (12) in the second case ( $\mathrm{K}$ is set to 0.25). CLMR attempts to detour the ideal path when parking with these values, and the inference is adjusted. Although fuzzy thresholds are higher in the sedan segment than in the hatchback segment, Fig. 14(a-i) shows CLMR entering parking (moving left) with the best route, so space is sufficient to travel through obstacles. 


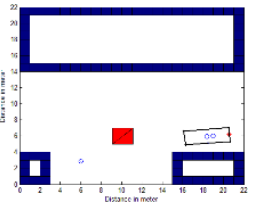

(a)

(d)

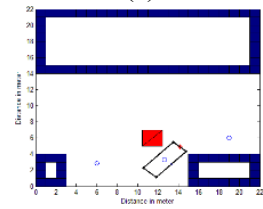

(g)

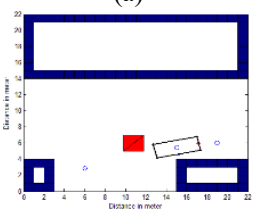

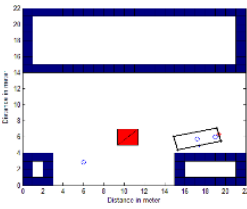

(b)

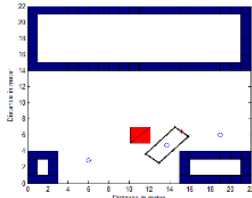

(e)

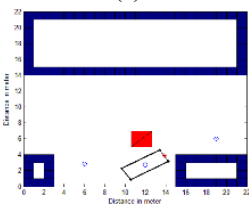

(h)

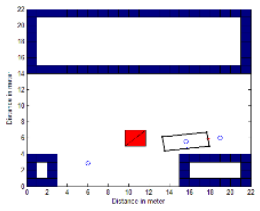

(c)

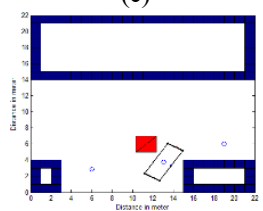

(f)

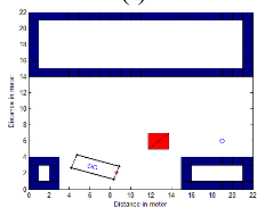

(i)
Fig. 14. The step sequence frames of reverse parking with $\mathrm{K}=0.25$

From the simulation results obtained for both vehicles, it can be inferred that scaling of the thresholds for fuzzy variables makes it possible for the car to pass through the narrow gaps and tight spaces. When the value of $\mathrm{K}$ is decreased, the minimum distance from the vehicle's body to the obstacle (dist_min) at any instant is also reduced. So there should always be a bottom limit for scaling down. In semi-automatic drive, the tuning of the scaling factor $\mathrm{K}$ can significantly improve the vehicle's path with expert driver inference from the environment. Simulation results for other passenger vehicles are summarized in table 2 . The selection of $\mathrm{K}$ is heuristically set to 0.25 for the dataset and kept above the bare minimum thresholds. Kmax in table 2 shows the upper threshold of selecting $\mathrm{K}$ with allowing the vehicle to pass the narrow gaps. Any value higher than K may not result in the car passing through tight spaces.

Table 2 Minimum safe distance achieved for a different vehicle in the simulation

\begin{tabular}{cccccc}
\hline \hline $\begin{array}{c}\text { Passenger } \\
\text { vehicle }\end{array}$ & Length(mm) & Width(mm) & Kmin & Kmax & $\begin{array}{c}\text { Dist_min } \\
(\mathrm{mm}) \\
(\text { K-0.25) }\end{array}$ \\
\hline Nano & 3164 & 1495 & 0.24 & 0.47 & 238 \\
Datsun & 3429 & 1560 & 0.23 & 0.45 & 247 \\
Go & & & & & \\
Alto & 3430 & 1515 & 0.22 & 0.44 & 255 \\
WagonR & 3599 & 1475 & 0.20 & 0.42 & 275 \\
i10 & 3585 & 1595 & 0.22 & 0.44 & 253 \\
polo & 3987 & 1698 & 0.21 & 0.43 & 264 \\
Swift & 3840 & 1735 & 0.23 & 0.45 & 249 \\
i20 & 3985 & 1734 & 0.22 & 0.44 & 259 \\
Honda & 4549 & 1748 & 0.19 & 0.44 & 293 \\
City & & & & & \\
Dzire & 3995 & 1735 & 0.22 & 0.43 & 259 \\
Verna & 4440 & 1729 & 0.19 & 0.44 & 289 \\
Amaze & 3995 & 1695 & 0.21 & 0.42 & 265 \\
Tigor & 3993 & 1677 & 0.21 & 0.42 & 268 \\
Civic & 4656 & 1799 & 0.19 & 0.44 & 291 \\
Creta & 4300 & 1790 & 0.21 & 0.42 & 270 \\
Fortuner & 4795 & 1855 & 0.19 & 0.44 & 291 \\
Harrier & 4598 & 1894 & 0.21 & 0.42 & 273 \\
XUV500 & 4585 & 1890 & 0.21 & 0.42 & 273 \\
& & & & &
\end{tabular}

$\begin{array}{cccccc}\text { Range } & 5200 & 2220 & 0.21 & 0.43 & 264 \\ \text { Rover } & & 1874 & 0.19 & 0.45 & 296 \\ \text { Audi A6 } & 4933 & 1811 & 0.19 & 0.45 & 300 \\ \text { BMW 3 } & 4824 & 2140 & 0.22 & 0.43 & 260 \\ \text { Volvo } & 4950 & 2126 & 0.22 & 0.43 & 261 \\ \text { XC90 } & & 1995 & 0.20 & 0.47 & 281 \\ \text { BMW 5 } & 4936 & 1948 & 0.18 & 0.44 & 312 \\ \text { Audi Q8 } & 4986 & & & & \\ \text { Rolls } & 5399 & & & \end{array}$

\section{CONCLUSION}

This paper aims to provide a solution to the two problems that autonomous parking systems face. First, it incorporates obstacle avoidance-based navigation intelligence into a standalone parking controller to handle a dynamic environment of moving and stationary obstacles. It achieves target steering to the parking due to a navigation module's inclusion, even though the car is momentarily detoured. Second, the obstacle avoidance module is kept adaptive for the passengers' vehicle dynamics to make this system suit every vehicle segment, regardless of its curvature. This algorithm is suitable for nonholonomic vehicles to navigate across small gaps and corners. The vehicle's route planning is greatly affected by choice of $\mathrm{K}$. It concludes that the value of $\mathrm{K}$ should be chosen lower when available space is limited and higher when space is not a restriction.

\section{References}

[1] C. J. Harris and C. G. Moore, "Intelligent identification and control for autonomous guided vehicles using adaptive fuzzy-based algorithms," Eng. Appl. Artif. Intell., vol. 2, no. 4, pp. 267-285, 1989, doi: 10.1016/0952-1976(89)90013-4.

[2] F. Gómez-Bravo, F. Cuesta, and A. Ollero, "Parallel and diagonal parking in nonholonomic autonomous vehicles," Eng. Appl. Artif. Intell., vol. 14, no. 4, pp. 419-434, 2001, doi: 10.1016/S0952-1976(01)00004-5.

[3] S. J. Chang and T. H. S. Li, "Design and implementation of fuzzy parallel-parking control for a car-type mobile robot," J. Intell. Robot. Syst. Theory Appl., vol. 34, no. 2, pp. 175-194, 2002, doi: 10.1023/A:1015664327686.

[4] T.-H. S. Li and Shih-Jie Chang, "Autonomous fuzzy parking control of a car-like mobile robot," IEEE Trans. Syst. Man, Cybern. - Part A Syst. Humans, vol. 33, no. 4, pp. 451-465, Jul. 2003, doi: 10.1109/TSMCA.2003.811766.

[5] T. H. S. Li, S. J. Chang, and Y. X. Chen, "Implementation of human-like driving skills by autonomous fuzzy behavior control on an FPGA-based car-like mobile robot," IEEE Trans. Ind. Electron., vol. 50, no. 5, pp. 867-880, 2003, doi: 10.1109/TIE.2003.817490.

[6] I. Baturone, F. J. Moreno-Velo, S. Sánchez-Solano, and A. Ollero, "Automatic design of fuzzy controllers for car-like autonomous robots," IEEE Trans. Fuzzy Syst., vol. 12, no. 4, pp. 447-465, 2004, doi: 10.1109/TFUZZ.2004.832532. 
[7] Y. Zhao and E. G. Collins, "Robust automatic parallel parking in tight spaces via fuzzy logic," Rob. Auton. Syst., vol. 51, no. 2-3, pp. 111-127, 2005, doi: 10.1016/j.robot.2005.01.002.

[8] K. Demirli and M. Khoshnejad, "Autonomous parallel parking of a car-like mobile robot by a neuro-fuzzy sensor-based controller," Fuzzy Sets Syst., vol. 160, no. 19, pp. 2876-2891, 2009, doi: 10.1016/j.fss.2009.01.019.

[9] C.-K. Lee, C.-L. Lin, and B.-M. Shiu, "Autonomous Vehicle Parking Using Hybrid Artificial Intelligent Approach," J. Intell. Robot. Syst., vol. 56, no. 3, pp. 319-343, Oct. 2009, doi: 10.1007/s10846-009-9319-9.

[10] T. H. S. Li, Y. C. Yeh, J. Da Wu, M. Y. Hsiao, and C. Y. Chen, "Multifunctional intelligent autonomous parking controllers for carlike mobile robots," IEEE Trans. Ind. Electron., vol. 57, no. 5, pp. 1687-1700, 2010, doi: 10.1109/TIE.2009.2033093.

[11] B. PANOMRUTTANARUG and K. HIGUCHI, "Fuzzy Logic Based Autonomous Parallel Parking System with Kalman Filtering," SICE J. Control. Meas. Syst. Integr., vol. 3, no. 4, pp. 266-271, 2010, doi: 10.9746/jcmsi.3.266.

[12] S.-J. Huang and Y.-S. Hsu, "Parking Space Detection And Trajectory Tracking Control For Vehicle AutoParking," Int. J. Mech. Mechatronics Eng., vol. 11, no. 10, pp. 1712-1718, 2017, [Online]. Available: https://login.e.bibl.liu.se/login?url=https://search.ebsc ohost.com/login.aspx?direct=true\&AuthType=ip,uid\& $\mathrm{db}=$ edsbas\&AN=edsbas.F64BA561\&amp\%0Alang=s $\mathrm{v} \&$ site $=$ eds-

live\&scope $=$ site $\% 0$ Ahttps://login.e.bibl.liu.se/login?ur l=https://search.ebscohost.com/login.aspx?d.

[13] C. Li, H. Jiang, S. Ma, S. Jiang, and Y. Li, "Automatic parking path planning and tracking control research for intelligent vehicles," Appl. Sci., vol. 10, no. 24, pp. 127, 2020, doi: 10.3390/app10249100.

[14] K. Wu, M. Karkoub, and T. Wu, "Robust adaptive fuzzy control design for 3-D tower crane with time delayed states," Int. Conf. Syst. Control Informatics, vol. 2, pp. 193-200, 2013, [Online]. Available: http://europment.com/library/2013/venice/bypaper/SC I/SCI-29.pdf.

[15] W. Li, "Fuzzy-logic-based reactive behavior control of an autonomous mobile system in unknown environments," Eng. Appl. Artif. Intell., vol. 7, no. 5, pp. 521-531, 1994, doi: 10.1016/0952-1976(94)900310.

[16] C. M. Johnson and J. O. Gray, "Hierarchical pathplanning for a mobile robot," Eng. Appl. Artif. Intell., vol. 7, no. 2, pp. 137-149, Apr. 1994, doi: 10.1016/0952-1976(94)90018-3.

[17] W. L. Xu and S. K. Tso, "Sensor-based fuzzy reactive navigation of a mobile robot through local target switching," IEEE Trans. Syst. Man Cybern. Part C Appl. Rev., vol. 29, no. 3, pp. 451-459, 1999, doi: 10.1109/5326.777079.

[18] G. Antonelli, S. Chiaverini, and G. Fusco, "A fuzzylogic-based approach for mobile robot path tracking," IEEE Trans. Fuzzy Syst., vol. 15, no. 2, pp. 211-221,
2007, doi: 10.1109/TFUZZ.2006.879998.

[19] C. L. Hwang and C. Y. Shih, "A distributed activevision network-space approach for the navigation of a car-like wheeled robot," IEEE Trans. Ind. Electron., vol. 56, no. 3, pp. 846-855, 2009, doi: 10.1109/TIE.2008.2004388.

[20] M. M. Joshi and M. A. Zaveri, "Fuzzy based autonomous robot navigation system," Proc. INDICON 2009 - An IEEE India Counc. Conf., 2009, doi: 10.1109/INDCON.2009.5409419.

[21] M. A. K. Jaradat, M. H. Garibeh, and E. A. Feilat, "Autonomous mobile robot dynamic motion planning using hybrid fuzzy potential field," Soft Comput., vol. 16, no. 1, pp. 153-164, 2012, doi: 10.1007/s00500-0110742-z.

[22] T. Y. Abdalla, A. A. Abed, and A. A. Ahmed, "Mobile robot navigation using PSO-optimized fuzzy artificial potential field with fuzzy control," J. Intell. Fuzzy Syst., vol. 32 , no. 6 , pp. 3893-3908, 2017, doi: 10.3233/IFS162205.

[23] C. Y. Chou and C. F. Juang, "Navigation of an autonomous wheeled robot in unknown environments based on evolutionary fuzzy control," Inventions, vol. 3, no. 1, 2018, doi: 10.3390/inventions3010003.

[24] C. H. Chao, C. H. Ho, S. H. Lin, and T. H. S. Li, "Omnidirectional vision-based parallel-parking control design for car-like mobile robot," Proc. 2005 IEEE Int. Conf. Mechatronics, ICM '05, vol. 2005, pp. 562-567, 2005, doi: 10.1109/ICMECH.2005.1529319.

[25] W. C. Wang and R. Chen, "A vision-based fuzzy logic controller for backing-up an autonomous vehicle," $J$. Intell. Fuzzy Syst., vol. 19, no. 4-5, pp. 273-284, 2008. [26] Y. Y. Aye, K. Watanabe, S. Maeyama, and I. Nagai, "Design of an image-based fuzzy controller for autonomous parking of four-wheeled mobile robots," Int. J. Appl. Electromagn. Mech., vol. 52, no. 3-4, pp. 859-865, 2016, doi: 10.3233/JAE-162180.

[27] J. Van Brummelen, M. O'Brien, D. Gruyer, and H. Najjaran, "Autonomous vehicle perception: The technology of today and tomorrow," Transp. Res. Part C Emerg. Technol., vol. 89, no. July 2017, pp. 384-406, 2018, doi: 10.1016/j.trc.2018.02.012.

[28] D. Barth, I. A. Gorlach, P. Elizabeth, and S. Africa, "Modelling of a Thermal Spraying Controller Using MATLAB / Simulink," vol. 2, pp. 392-397, 2013.

[29] P. Kumar, H. Mohan, and G. A. Hoshoudy, "FLR Effect on Stability of a Plasma in Porous Medium," vol. 2, pp. 90-97, 2020.

[30] N. Nakrani and M. Joshi, "Fuzzy based Autonomous Parallel Parking Challenges in Real time Scenario," in Advances in Intelligent Systems and Computing, 2016, pp. $789-802$.

[31] N. Nakrani and M. Joshi, "An Intelligent Fuzzy based Hybrid Approach for Parallel Parking in Dynamic Environment," Procedia Comput. Sci., vol. 133, pp. 82-91, 2018, doi: 10.1016/j.procs.2018.07.011. 


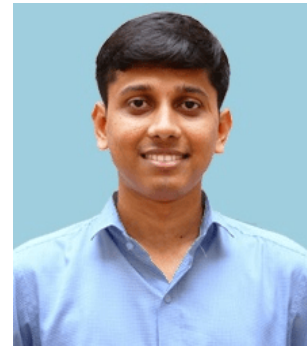

Naitik M. Nakrani is a Ph.D. scholar working in the field of Robotics and Machine learning. He has received B.E. in Electronics \& Communication from the Dharmsinh Desai University, Nadiad, in 2010, and received M.E. in Electronics \& Communication from Gujarat Technological University in 2012. He has worked as an Assistant professor in Electronics and Communication dept, UTU, for eight years. His research interests are autonomous systems, robotics, machine learning, fuzzy system, etc. He is an IEEE member since 2019 and served as a branch counselor of IEEE SB Uka Tarsadia University. He is also a lifetime member of ISTE and I.E.

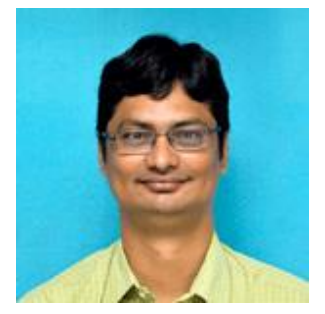

Dr. Maulin Joshi is currently serving as a Professor and head at Sarvajanik College of Engineering and Technology, Surat. He completed his B.E. from DDIT Nadiad in 1996, Masters from IIT Bombay in 2005, and Ph.D. from SVNIT in 2012. He has 22 years of teaching experience at the U.G. and P.G. level and 17 years of research experience. He is a regular contributor to various peer-reviewed journals and conferences, serves on various program committees, and a reviewer for reputed journals. He is SMIEEE currently volunteering as a Technical activity chair at the IEEE Gujarat section. He is a fellow to IETE and a senior member of CSI and has served as a Surat region chair in both.

\section{Creative Commons Attribution License 4.0 (Attribution 4.0 International, CC BY 4.0)}

This article is published under the terms of the Creative Commons Attribution License 4.0

https://creativecommons.org/licenses/by/4.0/deed.en_US 